\title{
Influence of Measuring Points in Ultrasonic Tests on the Tomographic Image Quality of Concrete
}

\author{
Marinara A. N. Moura \\ University of Campinas \\ R. Paschoal Marmo, \\ 1888 \\ Brazil 13484-350, \\ Limeira, SP \\ m228048@dac.unicamp.br
}

\author{
Marco A. G. Carvalho \\ University of Campinas \\ R. Paschoal Marmo, \\ 1888 \\ Brazil 13484-350, \\ Limeira, SP \\ magic@ft.unicamp.br
}

\author{
Gisleiva C. S. Ferreira \\ University of Campinas \\ R. Paschoal Marmo, \\ 1888 \\ Brazil 13484-350, \\ Limeira, SP \\ gisleiva@ft.unicamp.br
}

\begin{abstract}
In the process of creating digital images of concrete, wave propagation can be used to generate a suitable inner representation of this element, producing an ultrasonic tomography. Although tomography devices are efficient in generating images, they are high-cost and not very accessible. On the other hand, images obtained through ultrasound tests allow identifying the presence of flaws in different types of materials at lower costs. In ultrasonic tests, ultrasonic wave velocity paths are generated from different measurement points. Thus, in order to improve the data range, interpolation techniques can be used to estimate the acoustic velocity at the unmeasured test points, completing the image creation process. This work aims to evaluate the influence on the quality of the generated image of (1) the spatial resolution and (2) the number of measurement points in the ultrasonic test. Experiments were carried out using Inverse Distance Weighting interpolation technique and ultrasonic tomography on concrete beams with and without flaws. The resulting images indicated the importance of the appropriate choice of number and arrangement of measuring points in the ultrasonic test to properly detect internal flaws. In addition, we verify that a higher spatial resolution may also interfere in the identification of internal flaws in the image.
\end{abstract}

\section{Keywords}

Concrete, Ultrasonic Test, Ultrasonic Tomography, Data Interpolation.

\section{INTRODUCTION}

Concrete is the most used building material. Several structures, such as Bridges, Buildings, Structures, among others, are continuously subject to the effects that degrade their structural integrity [MEH06]. For this reason, it is necessary to take preventive actions to inspect and monitor the concrete, to ensure the safety of the structures and maintain their design performance.

It is usual to notice internal flaws in reinforced concrete construction elements, due to the execution failures or curing problems. Among the most common flaws are the concreting voids, which are difficult to identify and locate and can cause harmful consequences to structures. The difficulties on identifying and locating this flaw can be managed by using non-destructive inspection methods. The Non-Destructive Testing (NDT) is a

\footnotetext{
Permission to make digital or hard copies of all or part of this work for personal or classroom use is granted without fee provided that copies are not made or distributed for profit or commercial advantage and that copies bear this notice and the full citation on the first page. To copy otherwise, or republish, to post on servers or to redistribute to lists, requires prior specific permission and/or a fee.
}

technique used to evaluate a material without damaging it.

Among the advantages of NDT, it allows controlling the concrete quality in new constructions, evaluate the conditions of existing structures and also ensure the quality of repair work. In addition, NDT are used to assess the uniformity of the material, the modulus of elasticity and strength of concrete [CAR19].

The ultrasonic test uses the wave propagation to represent the flat section of the concrete in a $2 \mathrm{D}$ image. This technique has been widely used in the inspection field because it is portable, non-invasive and low-cost. In fact, the test is the most reliable, easy and portable nondestructive method used for assessing elastic properties of materials [HAS12].

Several studies have discussed the use of ultrasonic tests for detecting internal flaws in concrete. Parameters such as the number of measuring points in the test can significantly influence the results, discussed by [HAA16] and [PER19].

By means of the interpolation technique it is possible to reconstruct or approximate the continuous signals from a series of discrete points [CHE19]. This technique has been widely applied to super-resolution and im- 
age reconstruction in ultrasonic tomography [DON16] [FAN17] [FAN18].

This study intends to evaluate the influence of the number of measured points in the ultrasonic test and, consequently, on the tomography image quality generated after the interpolation process. The influence of the resolution will also be evaluated on the quality of the generated image. The interpolation method used to generate the images was the Inverse Distance Weighting (IDW).

The reminder of this paper is organized as follows: In Section 2 we address related work and basic concepts. The proposed method, the fundamentals and details of each step in the method are addressed in Section 3. The experiments and results are shown in Section 4. Finally, in Section 5 we presented our conclusions.

\section{RELATED WORK AND FUNDA- MENTAL CONCEPTS}

This section introduces some basic concepts of Ultrasonic Tomography and Data Interpolation. Furthermore, some of related known methods are discussed.

\subsection{Fundamental Concepts of Ultrasonic Tomography}

Ultrasonic tomography is an NDT method that allows the mapping of the entire internal section of the object from its projections of ultrasonic waves. In contrasting with traditional ultrasonic single measurement techniques, tomographic ultrasound technique provides methods of sampling a substantial volume rather than a single point, with an automated way of data collection and processing [KOU19]. It provides visualization, either by the cross section or by the three-dimensional structure. A better identification of the anomalous regions and determination of the physical properties of the measured region can be obtained [BON00].

Recently, ultrasound tomography has attracted a great deal of interest in a wide spectrum of applications. In the industrial field, a study demonstrated the effectiveness of an online sound speed travel-time ultrasound computed tomography system to inspect liquid mixtures of different densities. The study shows the soundspeed tomographic imaging to monitor liquid elaboration processes [KOU19]. In the biological field, the development of multi-spectral optoacoustic tomography techniques was proposed by [MER19] for whole-body computed tomography of mice, in a devised a hybrid transmission-reflection optoacoustic ultrasound (TROPUS) imaging platform. The method proved that the noninvasive technique of ultrasound imaging can also provides highly complementary information on elastic and functional tissue properties of small animals, enhancing optoacoustic image quality.In additional,
Although efficient tomography devices are already available, the ultrasound test is more cost-effective and can produce even better images. Regarding to the ultrasonic test, it is performed by positioning the transmitting transducer and the receiver transducer, of the ultrasonic equipment, either side of the specimen as shown in Figure 1. The transmitting sends a compression wave through the specimen, and the receiver records the full waveform on the other side. The time the wave takes to propagate through the specimen is registered, i.e., the Time Of Flight (TOF). In addition to the direct measurements, the test can be performed with the transducers on the same surface (indirect measurements) or on adjacent surfaces (semi-direct measurements), according to the American Standard ASTM-C597. [AST02].

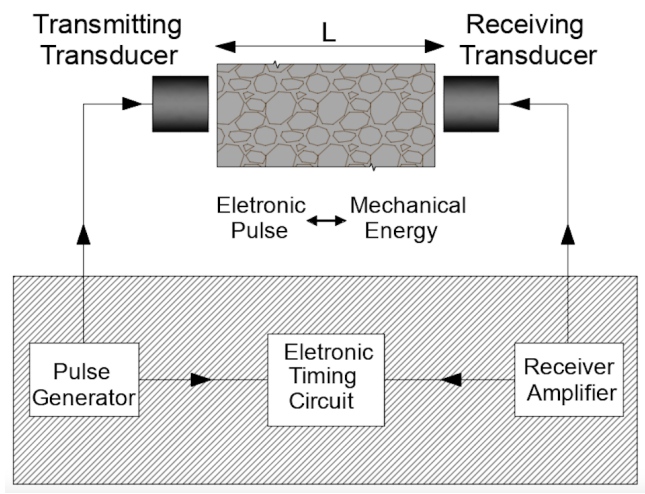

Figure 1: Ultrasonic Test on a Concrete Specimen of length $\mathrm{L}$.

In the ultrasonic reconstruction of a homogeneous material, the mechanical waves propagate in straight lines between the transducers. However, in a concrete specimen, the mechanical waves may deviate from an internal flaw, affecting the resulting tomography's [PER19].

\subsection{Fundamental Concepts of Data Inter- polation}

In an ideal interpolation, given the appropriate conditions, a continuous signal can be exactly reconstructed from the discrete points [CHE19]. A spatial interpolation can predict the values of the unknown points by processing the surrounding variables with meaningful values within the same region. This is often used when the measured data needs to be transformed from discrete points to a continuous data surface. [FEN14]

Based on the assumption that stress wave propagates as straight lines in the cross section of a tree, [FEN14] presented a study to investigate the effectiveness of using a proposed interpolation method to reconstruct a tomographic image from stress wave TOF data. The main hypothesis was that every point in the cross section of a tree trunk was related to other points and the adjacent points were more closely related than distant points. 
Their results included a good match found between tomographic images and the true physical conditions of the samples.

In concrete, the use of interpolation for generation and reconstruction of images is still not widely discussed. However, some studies presents the advantage of the method applied to concrete. In the study proposed by [FAN17], the maximum likelihood expectation maximization algorithm and ultrasonic TOF data was used to reconstruct concrete images. In order to obtain a high quality image, TOF data interpolation and normalization were proposed and validated for image reconstruction system. In the study conducted by [GOM19] three spatial interpolation techniques for generating digital concrete images were used: Nearest Neighbor Interpolation, IDW and a modified IDW technique. The results showed that the presence of voids in concrete could be identified using the IDW interpolation technique.

\subsection{Ultrasonic Tomography on Concrete}

The ultrasonic test has been effectively used for the evaluation of several types of concrete internal flaws, such as void detection [DON16] and cracking on concrete [YAN19]. A successful example of implementation was achieved on the evaluation of structures repair by comparing the pulse velocity of the structures before and after repair [AGG11]. Regarding the identification of non-homogeneity in concrete, [SCH14] used ultrasonic tomography to determine the location and size of internal flaws in a sample of concrete and confirmed the reliability of the technique. In the experiment proposed by [FAN18], the images of concrete specimens were reconstructed by using transit time parameters interpolation and MLEM improved algorithm. The proposed image reconstruction technique was effective to improve the accuracy and the quality of image reconstruction on concrete. [HAA16] studied the influence of different number of measurements in the ultrasonic test. Using the principle of image reconstruction, their tomographic images were generated using the transformation of each TOF data as sums of partial traveltimes in all elements of a defined mesh. The Simultaneous Iterative Reconstruction Technique (SRIT) algorithm was used to solve the system of linear equations of all measurements generated. The results demonstrated that quality of the tomographic images depends on the number of the measurements and its distribution. [PER19] Also performed ultrasonic tests for detecting flaws in tomographic images of concrete. They used the Network Theory to improve the ultrasonic tomography in concrete. This theory uses a network of interconnected nodes, defining the permissible ultrasonic travel paths. By dividing the object into elements, each element was added to secondary nodes. The Dijkstra's algorithm is used to identify which travel path is the fastest. Then, for the principle of image reconstruction, the SIRT technique is also applied to solve the system of linear equations. The authors found out that the reduction of the mesh size (greater number of measuring points) provided a better image than the increment of secondary nodes (network theory proposed by the authors), proving the importance of choosing an adequate mesh for generating tomographic image by means of ultrasonic test. The use of more robust interpolation techniques have received attention in the last years. On the other hand, simpler techniques can have computational advantages if they are efficient for what is being proposed. [GOM18] used the interpolation technique to detect concrete internal flaws. They used IDW interpolation, as one of techniques proposed achieved satisfactory results in the ultrasonic tomographic images.

Considering previous work, our contribution are mainly concerned in the use of basic interpolation techniques to generate digital images for detecting internal flaws in concrete, enabling the generation of tomographic images at lower computational cost. Besides, the study of resolution in image quality is also directly linked to computational cost.Finally, the study was conducted on two reinforced concrete beams of different configurations. Although many works use a large amount of concrete specimen in their studies, the use of a concrete beam containing steel armour is more linked to practice, bringing the situation closer to reality.

\section{PROPOSED APPROACH}

In this section we describe the proposed approach of this work. It consists of 4 steps which are illustrated in Figure 2 and described though the remainder of this section.

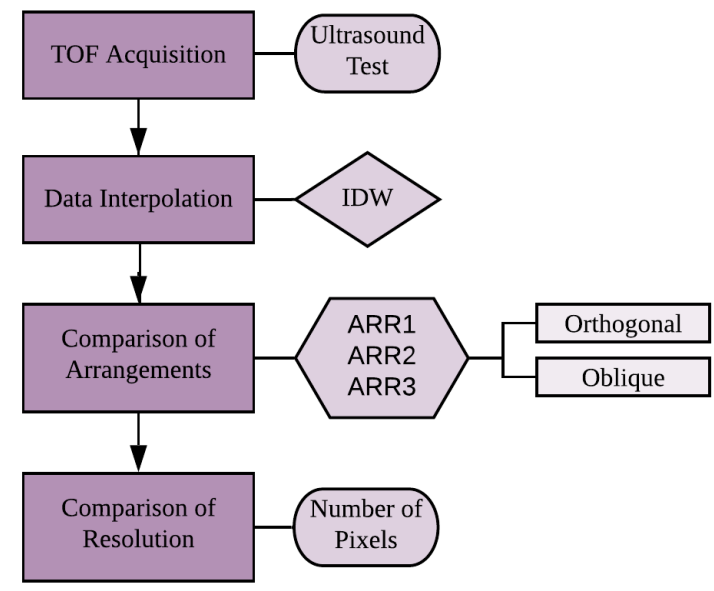

Figure 2: Workflow of proposed approach.

\subsection{TOF Acquisition}

The Time of Flight (TOF) acquisition consists in obtaining the ultrasonic pulse propagation times (TOF) by means of ultrasonic tests. The ultrasonic tests were 
performed on two reinforced concrete beams, 3 meters long, 0.4 meters high and 0.2 meters wide produced by [ROC17], both concreted with the same concrete. PVC pipes were included in one of the beams in order to simulate concreting voids defects. It had different sizes and locations according to Table 1. Figure 3(a) shows the longitudinal side of BEAM-A and the sections (1-1', 22', 3-3' and 4-4') used to generate the images with the TOF data. Figure 3(b) shows the longitudinal side of BEAM-B and the sections (A-A',B-B',C-C' and D-D') used to generate the images with the TOF data.

\begin{tabular}{|c|c|c|}
\hline Pipe & Diameter(mm) & Length(mm) \\
\hline 1 & 25 & 150 \\
\hline 2 & 50 & 200 \\
\hline 3 & 25 & 300 \\
\hline 4 & 75 & 100 \\
\hline
\end{tabular}

Table 1: PVC pipes dimensions.

The comparison will be carried out between the two sections of BEAM-B that contain PVC pipes, and the other that do not contain. Besides, the 4 cross sections of BEAM-A will also be compared with the sections of BEAM-B, specially the ones without flaws since they have to be similar regardless of whether the beam has flaws or not.

\subsection{Data Interpolation}

The data interpolation was used to complement the generation of tomographic images. The method selected to interpolate the data in this work was the inverse distance weighting (IDW). This method is one of the most frequently used deterministic models in spatial interpolation. Its general idea is based on the assumption that the value of a point out of sample is the weighted average of known values within the neighborhood, and the weights are inversely related to the distances between the prediction location and the sampled locations [LU08].

In the IDW technique, the points closer to the point to be interpolated have more influence on the interpolation, because they have smaller distances compared to the others [HAR16].

Equation (1) describes the formulation of this interpolation method.

The value at any point $P$ is a weighted average of values at the data point in which weighting is a function of distance to those points ([SHE68].

$$
u(x)=\frac{\sum_{i=1}^{N}(d i)^{p} u_{i}}{\sum_{i=1}^{N}(d i)^{p}}
$$

Where $x$ is an interpolated (arbitrary) point (data point). $X i$ is an interpolating (known) point. $d i$ is the Cartesian distance between $x$ and $x i(\mathrm{~d}[\mathrm{x}, \mathrm{xi}]) . N$ is the total number of known points used in interpolation. $p$ is a positive real number, called the power parameter. uirepresents the noise value at position $\mathrm{i}$,

Since the IDW model is simple and fast, the proposed method to accomplish this study is computationally efficient.

\subsection{Study of Arrangements}

The third step consists in comparing the images generated with different arrangements of measuring points with orthogonal and oblique measurements. The ultrasonic test was conducted in 4 cross sections of each reinforced beam as shown Figure 3. It was used three arrangements (ARR1, ARR2 and ARR3) in order to make a comparison between the number of measured points for evaluation of the generated image. ARR1 had nine measuring points, ARR2 had five, and ARR3 had 3 measuring points. Each arrangement was done in two configurations: (1) orthogonal direct measurements, and (2) oblique direct measurements.

Orthogonal Direct Measurements: In this configuration, the TOF data was collected by using orthogonal direct measurements (Figure 3). Arrangements 1, 2 and 3 were used. Figure 4 shows the images generated in the 3 arrangements of Orthogonal Direct Measurements.

Oblique Direct Measurement: In this configuration, the test was performed in oblique direct measurements. In ARR1 it was build a mesh with nine measuring points, generating 81 travel paths. In ARR2 the mesh had 5 reading points, generating 25 travel paths. In ARR3, the mesh had 3 reading points , totaling 9 travel paths. Figure 5 shows the three stages of this configuration.

\subsection{Study of Resolution}

This study also includes the analysis of the spatial resolution influence on image quality for flaw detection. Considering the same size, images with better spatial resolution have a larger number of pixels than those with lower spatial resolution. Considering the size of the beam, images with the following pixel dimensions were generated: 10x20 pixels, 20x40 pixels and 40x80 pixels. If the pixel size becomes larger than the size of a flaw, it is possible that this flaw is not visualized in the digital image. Also, if it is possible to visualize the flaws with a lower resolution, the computational cost is lower. Thus, 3 different resolutions will be analyzed to choose the one that offers more advantage.

\section{EXPERIMENTS AND RESULTS}

The tests were conducted by the through-transmission method (direct transmissions), according to the American Report on Nondestructive testing [ACI13], with Pundit Lab commercial instrument at Proceq Company. 

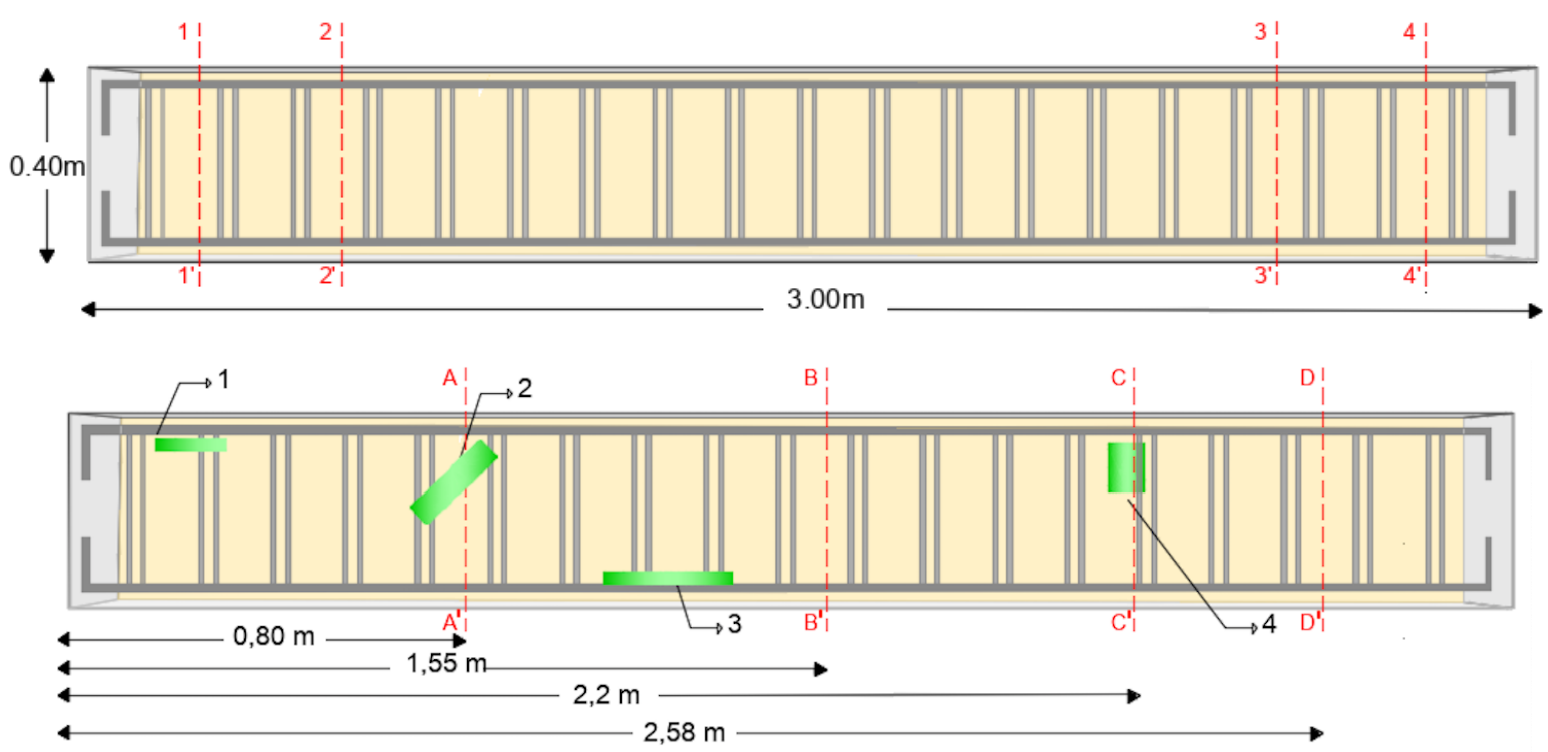

Figure 3: Reinforced Concrete Beam (a) without flaws and (b) with flaws. Adapted from [ROC17].

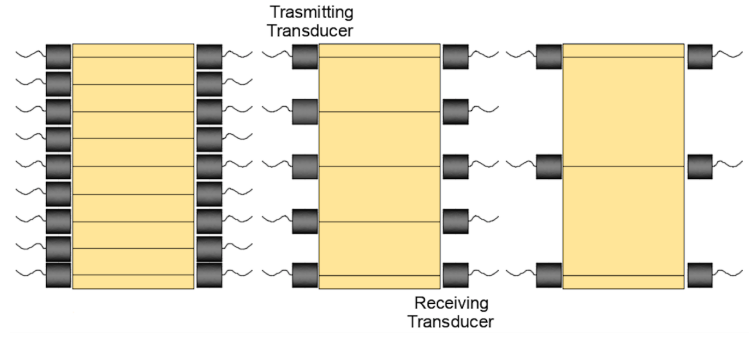

Figure 4: Cross Section of the beam in the three arrangements using Orthogonal Direct Measurements.

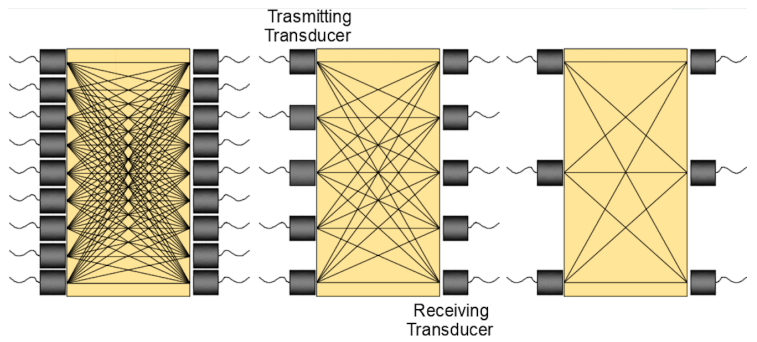

Figure 5: Cross Section of the beam in the three arrangements using Oblique Direct Measurements.

Transducers of longitudinal waves at $54 \mathrm{kHz}$ frequency were applied to the tests. The transducer face and the specimen were coupled by a medical ultrasound transmission gel. The images were generated using python's matplotlib library, in the "tab20c" color palette. In the IDW interpolation, the weight $(P)$ assigned to the distance between the points used was 2 .

\subsection{Study of Arrangements}

The images generated for the cross sections with orthogonal direct measurement are shown in Figures 6, 7 and 8 , for both BEAM-A and BEAM-B. The oblique direct measurements are represented in Figures 9,10 and 11 for BEAM-A and BEAM-B.
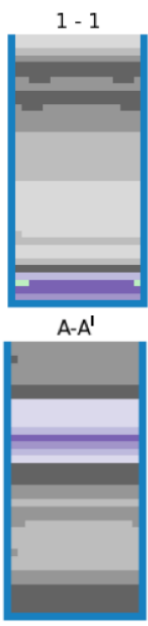

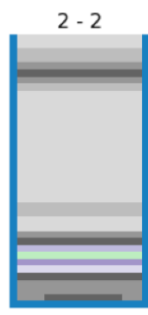

B - B'

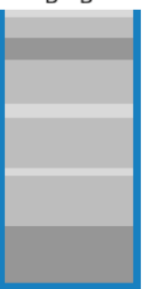

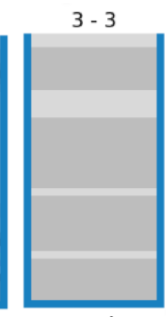

C - C

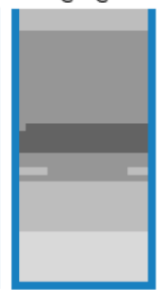

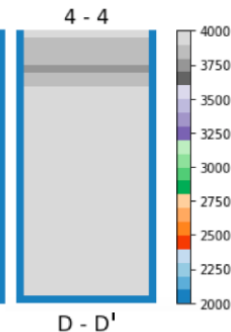

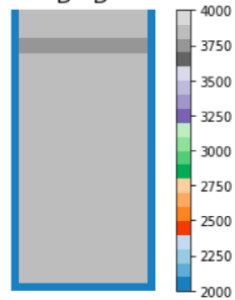

Figure 6: orthogonal direct measurements with 9 measuring points for (a) BEAM-A and (b) BEAM-B

In direct orthogonal measurements the images were generated with 1 pixel representing $1.0 \mathrm{~cm}^{2}$. The images generated from the sections without flaws (BEAM-A, and sections B-B' and D-D' of BEAM-B) are mostly homogeneous as shown in Figures 6,7 and 8 . In this configuration, section 1-1' and 2-2' did not show consistent results, as they are more alike to sections A-A' and C-C'(sections with flaws), where the non-homogeneity may be identified as a flaw in concrete, since the measurements at some points showed lower TOF. It is suggested that the results for direct measurements might be misleading, where non-homogeneous regions (with lower TOF values) could be interpreted as flaws even in sections where they do not occur, regardless of the number of measurements points. Also, the IDW technique may impact the sections without flaws, because it calculates 
$4-4$

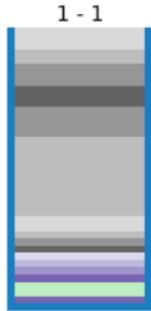

$A-A^{\prime}$

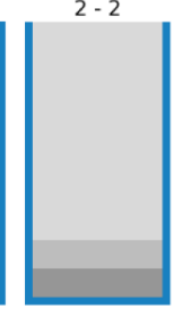

$B-B^{\prime}$
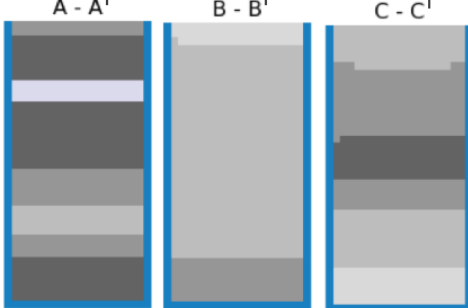

$3-3$
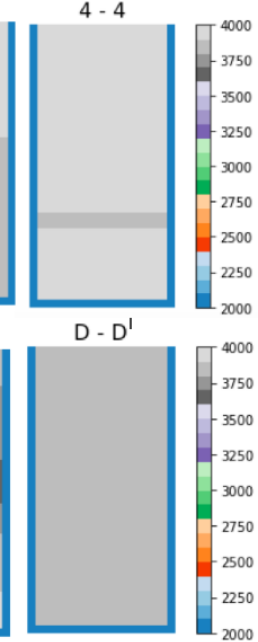

Figure 7: orthogonal direct measurements with 5 measuring points for (a) BEAM-A and (b) BEAM-B

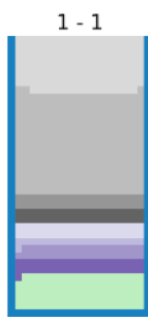

$A-A^{\prime}$
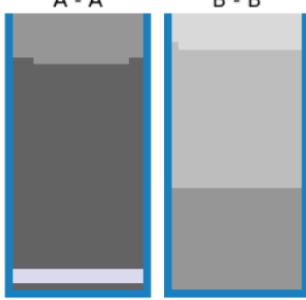

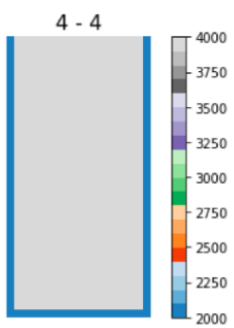

$D-D^{\prime}$
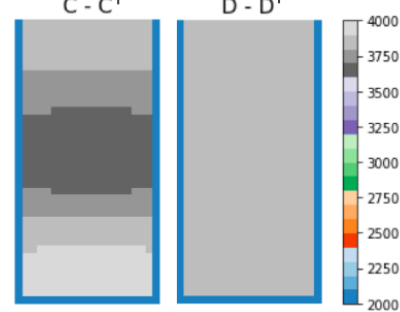

Figure 8: orthogonal direct measurements with 3 measuring points for (a) BEAM-A and (b) BEAM-B

the interpolated point considering all points measured in the section.

In direct oblique measurements the images were generated with 1 pixel representing $0.5 \mathrm{~cm}^{2}$. The flaws had more defined shapes, represented by the colors green and orange (lower TOF) according to Figures 9,10 and 11. As expected, the measurements with more travel paths presented better results ( 9 measuring points). However, anomalies could be identified even with 5 or 3 arrangements of measuring points.

Different from the orthogonal measurements, in oblique measurements, sections B-B 'and D-D' of BEAM-B resemble section 1-1 'of BEAM-A. Even though these are sections without flaws, some points with lower TOF values were identified. Even so, these sections are distinguished from sections A-A 'and C-C', where most of the image actually represents non-homogeneous regions identified by lower TOF values.
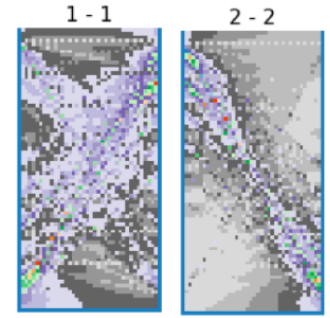

$A-A^{\prime}$

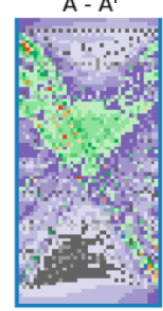

$B-B^{\prime}$

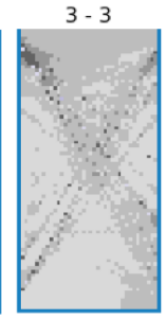

C - $\mathrm{Cl}^{\prime}$

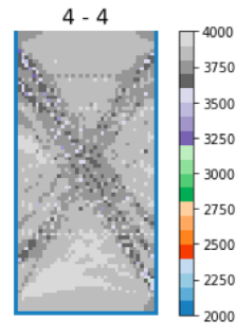

$D-D^{\prime}$
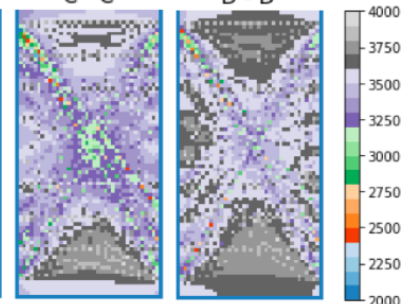

Figure 9: oblique direct measurements with 9 measuring points for (a) BEAM-A and (b) BEAM-B
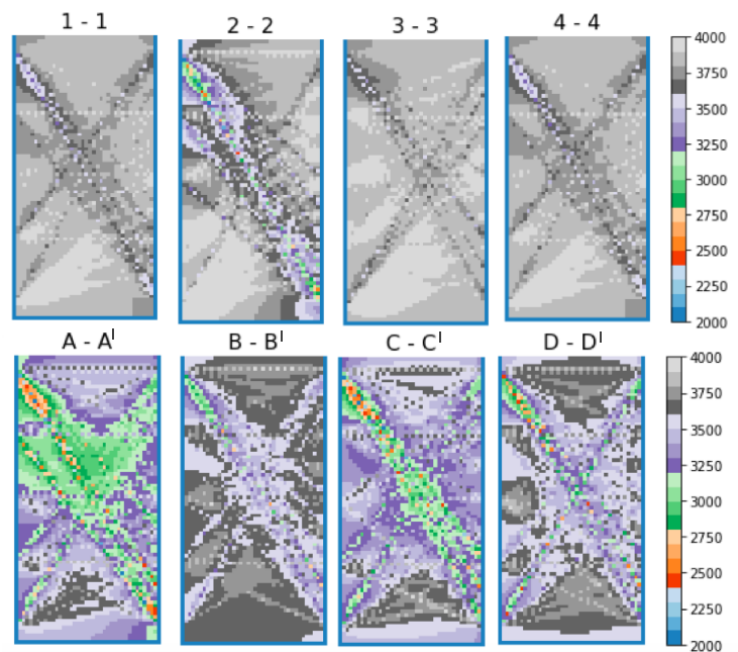

D - D
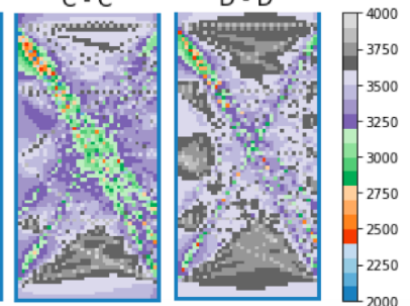

Figure 10: oblique direct measurements with 5 measuring points for (a) BEAM-A and (b) BEAM-B

The proposed algorithm only requires about $2.6 \mathrm{~s}$ to interpolate the images with 9 measurement points in oblique direct measurement (Figure 9), and it has much lower computational complexity than methods here commented. [HAA16] [FAN18][CHE19]

\subsection{Study of Resolution}

The resolution was analyzed by taking the sections with flaws (BEAM-B) as a reference, i.e., A-A' and C-C' in ARR2 (oblique direct measurements). A comparison was made among the images generated with $8 \times 4$ pixels, 20x10 pixels, 40x20 pixels and 80x40 pixels.

Figures 12 and 13 shows that the image generated with more pixels $(80 \times 40)$ presented better spatial resolution compared to the images generated with a smaller number of pixels (40x20 and 20x10). However, in a smaller number of pixels we understood that the presence of discontinuity could still be identified. In addition, when 


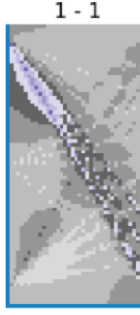

$A-A^{\prime}$
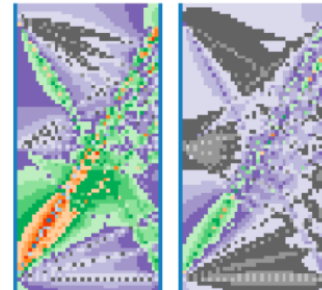

$B-B^{\prime}$

$3-3$
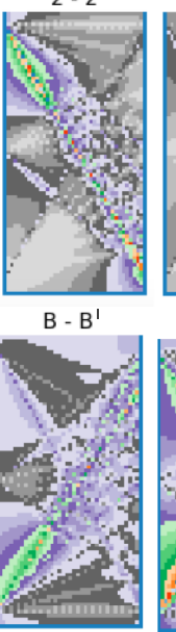

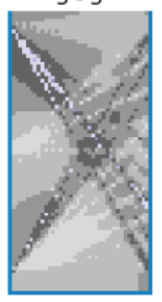

C - $C^{\prime}$

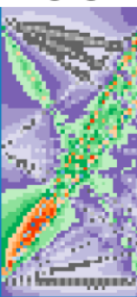

$4-4$

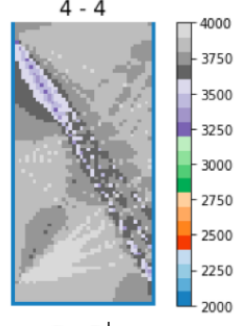

$D-D^{\prime}$

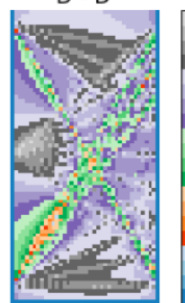

Figure 11: oblique direct measurements with 3 measuring points for (a) BEAM-A and (b) BEAM-B
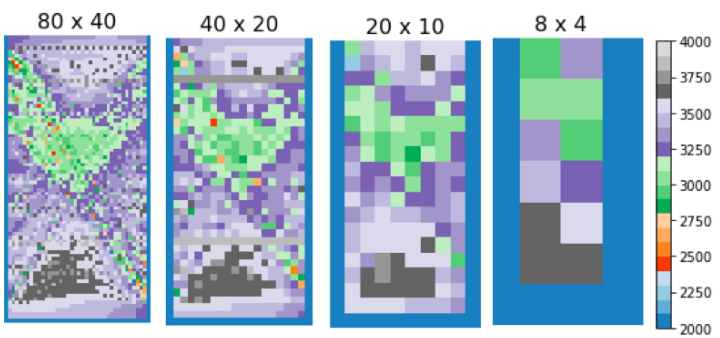

Figure 12: Image Generated with different resolutions for section A-A'.
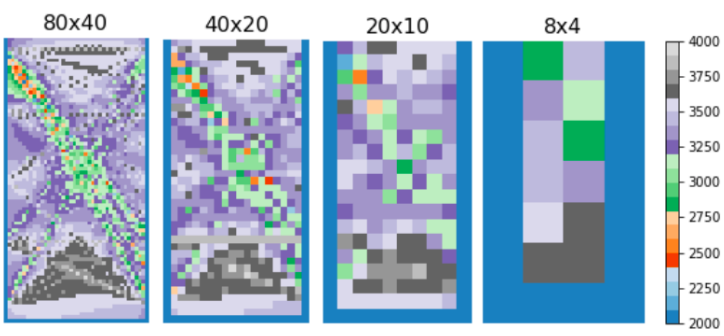

Figure 13: Image Generated with different resolutions for section $\mathrm{C}-\mathrm{C}$ '.

the pixel size becomes larger than the size of the smallest beam flaw $(8 \times 4)$, it is hard to detect any kind of flaw.

\section{CONCLUSIONS}

This paper presented a study of different arrangements of measuring points in the ultrasonic test and evaluate its influence on the result of the interpolation and image quality. The IDW technique was used to interpolate the data collected in the ultrasound test. The approach provided important information regarding the experimental procedures of ultrasonic tests. Also, some limitations and favorable conditions were identified. Our initial findings indicate that the quality of the images may depend on the number of measuring points. The direct orthogonal measurements did not show consistent results, since the images with and without flaws showed similar results. However, in the oblique measurements the presence of points that could represent a flaw in concrete, was identified even in arrangements of fewer measuring points. It should be noted that the IDW technique may influence the analysis of the sections without flaws, since it considers all points of the sample. Furthermore, the spatial resolution seemed to be more relevant than the number of measuring points. The tomographic images generated with more pixels in the direct oblique measurements achieved better results. Finally, ultrasonic tomography using data interpolation presented as a satisfactory technique for assessing concrete elements homogeneity. According to results, internal flaws in concrete were find considering different analysis. However, steel reinforcement, was not considered and may induce some errors. Future work includes the use of a larger database for verifying the results and the use of other type of interpolation. In addition, we intend to use image processing metrics in order to evaluate the results quantitatively.

\section{ACKNOWLEDGEMENTS}

This work was supported by Sao Paulo Research Foundation (FAPESP), grant 2018/18520-4.

\section{REFERENCES}

[ACI13] ACI-228.2R. Nondestructive test methods for evaluation of concrete in structures. American Concrete Institute Report, 2013.

[AGG11] Aggelis, D., Hadjiyiangou, S., Chai, H., Momoki, S., and Shiotani, T. Longitudinal waves for evaluation of large concrete blocks after repair. NDT \& E International, 44(1)pp.61-66, 2011.

[AST02] ASTM-C597. Standard Test Method for Pulse Velocity Through Concrete. American Society for Testing and Material, 2002.

[BON00] Bond, L. J., Kepler, W. F., and Frangopol, D. M. Improved assessment of mass concrete dams using acoustic travel time tomography. part i-theory. Construction and Building Materials, 14(3) pp. 133-146, 2000.

[CAR19] Carrillo, J., Ramirez, J., and LizarazoMarriaga, J.Modulus of elasticity and poisson's ratio of fiber-reinforced concrete in colombia from ultrasonic pulse velocities.Journal of Building Engineering, 23 pp. 18-26, 2019.

[CHE19] Cheng, D. and Kou, K. I. FFT multichannel interpolation and application to image super resolution. Signal Processing, 162:21-34, 2019.

[DON16] Dong, W., Wu, Z., Zhou, X., and Tan, Y. Experimental studies on void detection in concretefilled steel tubes using ultrasound. Construction and Building Materials, 128:154-162, 2016. 
[FAN17] Fan, H., Zhu, H., Zhao, X., Zhang, J., Wu, D., and Han,Q. Ultrasonic image reconstruction based on maximum likelihood expectation maximization for concrete structural information. Computers \& Electrical Engineering, 62:293-301, 2017.

[FAN18] Fan, H., Zhu, H. Improved image reconstruction based on ultrasonic transmitted wave computerized tomography on concrete. EURASIP Journal on Image and Video Processing, 2018:129,2018.

[FEN14] Feng, H., Li, G., Fu, S., and Wang, X. Tomographic image reconstruction using an interpolation method for tree decay detection. BioResources,v. 9, n. 2, p. 3248-3263, 2014.

[GOM18] Gomes, H. G. and Goncalves, A. Analyses of Interpolation Technique for generations of TomographicImages in Concrete. Undergraduated Work (System of Information), UNICAMP (University of Campinas),Limeira, Brazil, 2018.

[GOM19] Gomes, H. G., Goncalves, A.,Carvalho, M., Moura, M., Ferreira, G., Rocha A. Analyses of Interpolation Technique for generations of Tomographic Images in Concrete. ,In Proceedings of the 61 Concrete Conferece, IBRACON ,Fortaleza, Brazil, 2019.

[HAA16] Haach, V. G. and Ramirez, F. C. Qualitative assessment of concrete by ultrasound tomography. Construction and Building Materials, 119:61-70, 2016.

[HAR16] Harman, B. I., Koseoglu, H., and Yigit, C. O. Performance evaluation of IDW, kriging and multiquadric interpolation methods in producing noise mapping: Acase study at the city of isparta, turkey. Applied Acoustics, 112:147-157, 2016.

[HAS12] Hassan, A. and Jones, S. Non-destructive testing of ultra high performance fibre reinforced concrete (uh-pfrc): A feasibility study for using ultrasonic and resonant frequency testing techniques.Construction and Building Materials, 35:361-367, 2012.

[KOU19] Koulountzios, P., Rymarczyk, T., and Soleimani, M. A Quantitative Ultrasonic TravelTime Tomography to Investigate Liquid Elaborations in Industrial Processes. Sensors, v. 19, n. 23, p. $5117,2019$.

[LU08] Lu, G. Y. and Wong, D. W. An adaptive inverse distance weighting spatial interpolation technique. Computers \& geosciences, 34(9):10441055,2008 .

[MEH06] Mehta, P. K. and Monteiro, P. J. M. Concrete: Microstructure, Properties and Materials. McGraw-Hill,New York, 3rd edition, 2006.

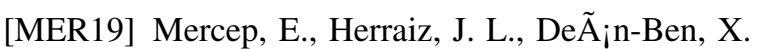

L., and Razansky, D. Transmission-reflection optoacoustic ultrasound (TROPUS) computed tomography of small animals. Light: Science \& Applications, v. 8, n. 1, p. 1-12, 2019.

[PER19] Perlin, L. P. and de Andrade Pinto, R. C. Use of network theory to improve the ultrasonic tomography in concrete. Ultrasonics, 96:185-195, 2019.

[ROC17] Rocha, A. I. B. C. Identification of Pathologies in Reinforced Concrete Beams Using Ultrasound Inspection. Master Thesis (Master of Technology),UNICAMP (University of Campinas), Limeira,Brazil, 2017.

[SCH14] Schabowicz, K. Ultrasonic tomography - the latest nondestructive technique for testing concrete members - description, test methodology, application example. Archives of Civil and Mechanical Engineering, 14(2):295-303, 2014.

[SHE68] Shepard, D. (1968). A two-dimensional interpolation function for irregularly-spaced data. In Proceedings of the 1968 23rd ACM national conference, pages 517-524.ACM, 1968.

[YAN19] Yang, C. and Chen, J. Fully noncontact nonlinear ultrasonic characterization of thermal damage in concrete and correlation with microscopic evidence of material cracking. Cement and Concrete Research, 123:105797-105807, 2019. 